November 2006

KUNS-2040

RIKEN-TH 83

\title{
Field Equations of Massless Fields in the New Interpretation of the Matrix Model
}

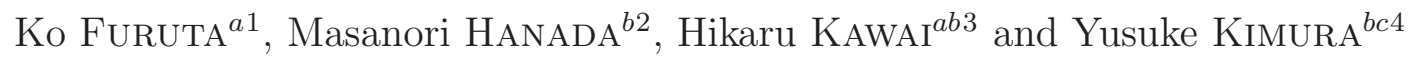 \\ a Theoretical Physics Laboratory, \\ The Institute of Physical and Chemical Research (RIKEN), \\ Wako, Saitama 351-0198, Japan \\ ${ }^{b}$ Department of Physics, Kyoto University, Kyoto 606-8502, Japan \\ ${ }^{c}$ Department of Physics, Queen Mary, University of London, \\ Mile End Road, London E1 4NS, England
}

\begin{abstract}
Recently, some of the authors have introduced a new interpretation of matrix models in which covariant derivatives on any curved space can be expressed by large- $N$ matrices. It has been shown that the Einstein equation follows from the equation of motion of IIB matrix model in this interpretation. In this paper, we generalize this argument to covariant derivatives with torsion. We find that some components of the torsion field can be identified with the dilaton and the $B$-field in string theory. However, the other components do not seem to have string theory counterparts. We also consider the matrix model with a mass term or a cubic term, in which the equation of motion of string theory is exactly satisfied.
\end{abstract}

\footnotetext{
1 E-mail address : furuta@riken.jp

2 E-mail address : hana@gauge.scphys.kyoto-u.ac.jp

3 E-mail address : hkawai@gauge.scphys.kyoto-u.ac.jp

4 E-mail address : y.kimura@qmul.ac.uk
} 


\section{Introduction}

String theory, as a candidate of unified theory of particle physics, is expected to provide knowledge of physics governing high-energy regime and possibly early time of the universe where the quantum effects of gravity become important. It is of crucial importance to have descriptions of string theory which give insight beyond perturbative level. Matrix models give efficient ways of approaching nonperturbative nature of string theory [1, 2]. In matrix models, how to describe the spacetime geometry has been one of key questions.

In the original proposal of matrix models, matrices have been considered as spacetime coordinates. In this description, the gravitational interaction appears as a loop effect, but the origin of the general covariance is unclear. In the recent paper [3], another interpretation of matrix models was proposed by some of the authors. It has been shown that a covariant derivative on any $d$ dimensional spacetime $M$ can be expressed by a set of $d$ operators that acts on the space of functions on the principal $\operatorname{Spin}(d)$ bundle over $M$. The Einstein equation follows from the equation of motion of the matrix model if we identify matrices with the covariant derivative in this way. Symmetries under local Lorentz transformation and diffeomorphism are manifestly realized as a part of the $U(N)$ symmetry. On the other hand, a general matrix should be regarded as a power series in the covariant derivative and Lorentz generator that contains infinitely many fields as coefficients. To understand their dynamics, it is important to investigate the degrees of freedom which appear around a classical solution. In this paper, we study the fields that appear as the coefficients of terms linear in the covariant derivative and Lorentz generator, that is, the vielbein and the torsion 5 . We discuss a possible interpretation of these fields in the framework of string theory.

The paper is organized as follows. In the next section, we review the new interpretation of matrix models [3]. In particular, we explain how the covariant derivative on a curved space can be expressed in terms of matrices. In section 3, we analyze the dynamics of the vielbein and the torsion. They form a closed subset of equations of motion and are expected to have consistent solutions without introducing higher spin fields. By identifying the trace and the antisymmetric parts of the torsion to be the field strengths of the dilaton and the antisymmetric $B$-field, respectively, we find solutions which resemble those in string theory. However, it turns out that ghost like degrees of freedom can also propagate in general. We might need to impose a constraint on the expansion of matrices. In section 4, we study modified models which have mass and cubic terms. In these cases, there are classical solutions with constant torsion. If we interpret the antisymmetric part of the torsion to be the field strength of the $B$-field, then these solutions satisfy the Einstein equation with a constant background of field strength of $B$. This supports the idea of embedding the $B$-field into the torsion that is introduced in section 3 . Section 5 is devoted to conclusions and discussions on future directions. Some of the details are contained in the appendices.

\footnotetext{
${ }^{5}$ Higher spin fields appear from coefficients of higher powers of covariant derivatives, and their gauge symmetries are included in the $U(N)$ symmetry [3]. Free equations of motion are also correctly reproduced from that of the matrix model [4.
} 


\section{New Interpretation of Matrix Model}

In this section, we briefly summarize the results obtained in [3]. We consider the large- $N$ reduced model of $d$-dimensional $U(N)$ Yang-Mills theory,

$$
S=-\frac{1}{4 g^{2}} \operatorname{Tr}\left(\left[A_{a}, A_{b}\right]\left[A^{a}, A^{b}\right]\right)
$$

where $A_{a}$ are $N \times N$ Hermitian matrices, and $a$ and $b$ run from 1 to $d$. (Supersymmetric version of this model with $d=10$ is known as IIB matrix model [2].) This model has the $U(N)$ gauge symmetry

$$
\delta A_{a}=i\left[\Lambda, A_{a}\right]
$$

where $\Lambda$ is a Hermitian matrix.

In [3], a new interpretation is proposed in which the matrices $A_{a}$ represent differential operators on a Riemannian manifold. In this interpretation, the symmetries of general relativity are contained naturally in the $U(N)$ symmetry, and the Einstein equation follows from the equation of motion of the matrix model.

To begin with, let us see how the covariant derivatives are described in terms of matrices. An important aspect of the covariant derivative is that it maps a rank- $n$ tensor to a rank- $(n+1)$ tensor. For example, $\nabla_{a} V_{b}$ is not the $a$-th component 6 of $\nabla$ acting on the $b$-th component of $V$, but is the $(a, b)$-component of a rank-two tensor $\nabla V$; indeed, in the formula

$$
\nabla_{a} V_{b}=e_{a}^{\mu}\left(\partial_{\mu} V_{b}+\omega_{\mu b}^{c} V_{c}\right)
$$

all of the components $V_{c}$ contribute to the covariant derivative. Here, $e_{a}{ }^{\mu}$ and $\omega_{\mu}{ }^{b c}$ are the vielbein and the spin connection, respectively.

Therefore, in order to express the covariant derivative in terms of matrices, the space on which matrices act must contain the tensors of all ranks. It was shown that the covariant derivative can be interpreted as a set of matrices (or equivalently, endomorphisms) acting on functions on the principal Spin $(d)$ bundle over the Riemannian manifold $M_{d}$. The space of functions on the group manifold $\operatorname{Spin}(d)$ forms the regular representation which contains tensors of all ranks. Then, the covariant derivative $\nabla_{a}$ is mapped to a set of matrices $\nabla_{(a)}$ by

$$
\begin{aligned}
\nabla_{(a)} & =R_{(a)}^{b}\left(g^{-1}\right) \nabla_{b} \\
& =R_{(a)}{ }^{b}\left(g^{-1}\right) e_{b}{ }^{\mu}(x)\left(\partial_{\mu}+\omega_{\mu}{ }^{c d}(x) \mathcal{O}_{c d}\right)
\end{aligned}
$$

where $g$ is the coordinate of the fiber $\operatorname{Spin}(d), R_{(a)}{ }^{b}\left(g^{-1}\right)$ is the vector representation of $\operatorname{Spin}(d)$ and $\mathcal{O}_{a b}$ is the Lorentz generator of the $\operatorname{Spin}(d)$ group. The operator $\nabla_{(a)}$ acts

\footnotetext{
${ }^{6} a, b, \cdots$ represent the Lorentz indices. We use $\mu, \nu, \cdots$ to express the Einstein indices.
} 
as endomorphism on the space of functions on the principal Spin $(d)$ bundle. Products of $\nabla_{(a)}$ are related to those of $\nabla_{a}$ as follows:

$$
\nabla_{(a)} \nabla_{(b)} \nabla_{(c)} \cdots=R_{(a)}^{a^{\prime}}\left(g^{-1}\right) R_{(b)}^{b^{\prime}}\left(g^{-1}\right) R_{(c)}{ }^{c^{\prime}}\left(g^{-1}\right) \nabla_{a^{\prime}} \nabla_{b^{\prime}} \nabla_{c^{\prime}} \cdots
$$

On the left hand side, each of the indices $(a),(b),(c)$ is nothing more than a label and there is no mixing of them in the product. Thus the product can be represented as the product of matrices $\nabla_{(a)}, \nabla_{(b)}, \nabla_{(c)}, \cdots$. On the right hand side, product of $\nabla_{a^{\prime}}, \nabla_{b^{\prime}}, \nabla_{c^{\prime}}, \cdots$ mixes the indices $b^{\prime}, c^{\prime}, \cdots$.

We regard that the matrices $A_{a}$ act on the space of functions on the principal Spin $(d)$ bundle over $M_{d}$. Then, $A_{a}$ can be expanded as series in powers of $\nabla_{(a)}$ and $\mathcal{O}_{a b}, 7$

$$
\begin{aligned}
A_{a}= & a_{(a)}(x, g)+\frac{i}{2}\left\{\delta_{(a)}{ }^{(b)}+b_{(a)}{ }^{(b)}(x, g), \nabla_{(b)}\right\}+\frac{i}{2}\left\{c_{(a)}{ }^{b c}(x, g), \mathcal{O}_{b c}\right\} \\
& +\frac{i^{2}}{2}\left\{d_{(a)}((b)(c))(x, g), \nabla_{(b)} \nabla_{(d)}\right\}+\frac{i^{2}}{4}\left\{\left\{e_{(a)}{ }^{b c(d)}(x, g), \mathcal{O}_{b c},\right\}, \nabla_{(d)}\right\}+\cdots .
\end{aligned}
$$

If we interpret the coefficients appearing in this expansion to be local fields, then the diffeomorphism, the local Lorentz transformation and higher-spin gauge transformations are contained naturally in the $U(N)$ symmetry of the matrix model [3, 4]. For example, the diffeomorphism is generated by $\Lambda=i \lambda^{(a)}(x) \nabla_{(a)}$. (We will show the explicit expression for the diffeomorphism and the local Lorentz transformation in section 3 , )

The equation of motion for the action (11) becomes

$$
\left[A^{a},\left[A_{a}, A_{b}\right]\right]=0 .
$$

As an ansatz, it is natural to require $A_{a}$ to be the differential operators of first-order, because their commutator is again of first-order and hence they form a closed algebra. Here, we consider the simplest possibility among them,

$$
A_{a}=i \nabla_{(a)} .
$$

(We consider a general ansatz in the next section.) Then, (7) becomes

$$
\left[\nabla^{a},\left[\nabla_{a}, \nabla_{b}\right]\right]=\left(\nabla^{a} R_{a b}^{c d}\right) \mathcal{O}_{c d}-R_{b}^{c} \nabla_{c}=0,
$$

where we have converted the indices $(a)$ and $(b)$ to the indices $a$ and $b$ using the equation (5). Because $\nabla^{a} R_{a b}{ }^{c d}=0$ follows from $R_{a b}=0$ and the Bianchi identity $\nabla^{[a} R_{a b}{ }^{c d]}=0$, the equation (9) reduces to $R_{a b}=0$ and thus provides the Einstein equation. Furthermore, if we regard the fields appearing in (6) to be fluctuations about the classical solution of the form (8), they can be interpreted as local fields propagating on the curved space $M_{d}$ which is represented by the covariant derivative $\nabla_{(a)}$; for example, $a_{a}(x)$ satisfies the Maxwell equation [3] and $d_{a}{ }^{b c}$ satisfies the free equations of motion for higher-spin fields [4]. Therefore, we can interpret that the classical solution derived above describes the Ricci-flat background spacetime. This is a new mechanism of the emergence of the spacetime in the matrix model.

7 The coefficients $a_{(a)}(x, g), b_{(a)}^{(b)}(x, g), \cdots$ are real-valued. We introduce the anticommutator $\{$,$\} in$ order to make $A_{a}$ Hermitian. Hereafter, we omit the anticommutator for simplicity. 


\section{Torsion as Massless Fields}

In this section, we consider the general form of the first-order differential operator, which is given by

$$
A_{a}=i D_{(a)} \equiv i R_{(a)}^{b}\left(g^{-1}\right) D_{b} \equiv i R_{(a)}^{b}\left(g^{-1}\right)\left(e_{b}^{\mu}(x) \nabla_{\mu}+S_{b}^{c d}(x) \mathcal{O}_{c d}\right)
$$

where $\nabla_{\mu}$ and $S_{a}^{b c}(x)$ are the torsionless covariant derivative and the contorsion, respectively 8 The differential operator $D_{a}$ can be regarded as a covariant derivative with torsion 9 As discussed in the previous section, because differential operators of first-order form a closed algebra, it is expected that the equation of motion has consistent solutions by setting all the fields in ([6) to be zero except for the fields appearing in the coefficients of (10). Note that it is possible to consider $g$-dependent fields $e_{b}{ }^{\mu}(x, g)$ and $S_{b}{ }^{c d}(x, g)$ in general. In this paper, we restrict our attention to the simplest case where $e_{b}{ }^{\mu}$ and $S_{b}{ }^{c d}$ has no $g$-dependence. For details concerning this point, see [3].

Before analyzing the equation of motion, it is helpful to study the gauge symmetries. The action (11) is invariant under the $U(N)$ transformation $\delta A_{a}=i\left[\Lambda, A_{a}\right]$, where $\Lambda$ is an $N \times N$ Hermitian matrix. If we restrict $\Lambda$ to be the first-order differential operator,

$$
\Lambda=i \lambda^{\mu}(x) \nabla_{\mu}+i \lambda^{a b}(x) \mathcal{O}_{a b}
$$

then the local fields appearing in (10) transform as

$$
\begin{aligned}
& \delta e_{a}{ }^{\mu}(x)=-\lambda_{a}{ }^{b}(x) e_{b}{ }^{\mu}(x)+\nabla_{a} \lambda^{\mu}(x), \\
& \delta \omega_{\mu}{ }^{b c}(x)=\nabla_{\mu} \lambda^{b c}(x)+\lambda^{\nu}(x) R_{\mu \nu}{ }^{b c}(x), \\
& \delta S_{a}{ }^{b c}=-\lambda^{\mu}(x) \nabla_{\mu} S_{a}{ }^{b c}(x)-\lambda_{a}{ }^{d} S_{d}{ }^{b c}(x)-\lambda^{b}{ }_{d} S_{a}{ }^{d c}(x)-\lambda^{c}{ }_{d} S_{a}{ }^{b d}(x) .
\end{aligned}
$$

Therefore, $i \lambda^{\mu}(x) \nabla_{\mu}$ and $i \lambda^{a b}(x) \mathcal{O}_{a b}$ generate the diffeomorphism and the local Lorentz transformation, respectively, and we can use them to remove unphysical modes of $e_{a}{ }^{\mu}(x)$. Then, no component of the contorsion $S_{a}^{b c}(x)$ can be gauged away.

Now, let us derive the equation of motion. The commutator of $D_{a}$ gives

$$
\left[D_{a}, D_{b}\right]=\mathcal{R}_{a b}^{c d} \mathcal{O}_{c d}+T_{a b}^{c} D_{c}
$$

where $T_{a b c}=S_{a b c}-S_{b a c}$ is the torsion and $\mathcal{R}_{a b}{ }^{c d}$ is the Riemann tensor with the contribution from the torsion, which is related to the ordinary Riemann tensor $R_{a b}{ }^{c d}$ by

$$
\mathcal{R}_{a b}{ }^{c d}=R_{a b}{ }^{c d}+\nabla_{a} S_{b}{ }^{c d}-\nabla_{b} S_{a}{ }^{c d}-S_{a e}{ }^{c} S_{b}{ }^{e d}+S_{b e}{ }^{c} S_{a}{ }^{e d} .
$$

\footnotetext{
${ }^{8}$ Although we can introduce coefficients of the covariant derivative as $A_{a}=i\left(a_{(a)}{ }^{(b)} \nabla_{(b)}+S_{(a)}{ }^{b c} \mathcal{O}_{b c}\right)$, such coefficients can be absorbed by the following field redefinition: $e_{(a)}^{\prime}{ }^{\mu}=a_{(a)}{ }^{(b)} e_{(b)}{ }^{\mu}, S_{(a)}^{\prime}{ }^{b c} \mathcal{O}_{b c}=$ $S_{a b c} \mathcal{O}_{b c}+\left(a_{(a)}^{(b)} \nabla_{(b)}-\nabla_{(a)}^{\prime}\right)$. Here $\nabla_{a}^{\prime}$ is the torsionless covariant derivative which is constructed from $e_{a}^{\prime \mu}$.

${ }^{9}$ The torsion gravity has been studied extensively as a generalization of general relativity, and it is interesting to clarify the relation of it to our approach. For reviews of the torsion gravity, see [5, 6].
} 
The equation of motion is given by

$$
\begin{aligned}
{\left[D^{a},\left[D_{a}, D_{b}\right]\right]=} & \left(-\mathcal{R}_{b}{ }^{c}+D^{a} T_{a b}{ }^{c}+T_{a b}{ }^{d} T^{a}{ }_{d}^{c}\right) D_{c} \\
& +\left(D^{a} \mathcal{R}_{a b}{ }^{c d}+T^{a}{ }_{b}{ }^{e} \mathcal{R}_{a e}{ }^{c d}\right) \mathcal{O}_{c d} \\
= & 0
\end{aligned}
$$

where $\mathcal{R}_{b c}=\mathcal{R}_{a b}{ }^{a}{ }_{c}$. Therefore, we obtain the following two equations:

$$
\begin{aligned}
& -\mathcal{R}_{b c}+D^{a} T_{a b c}+T_{a b}{ }^{d} T^{a}{ }_{d c}=0 \\
& D^{a} \mathcal{R}_{a b}{ }^{c d}+T^{a}{ }_{b}{ }^{e} \mathcal{R}_{a e}{ }^{c d}=0
\end{aligned}
$$

Note that (18) has not only the symmetric part, which can be regarded as the Einstein equation, but also the antisymmetric part. These equations are solved in the following subsections. We first analyze them at the linearized level in the next subsection. We find solutions in which torsion fields can be interpreted as the dilaton and the antisymmetric $B$-field in string theory. In subsection 3.2 we show that the equations have solutions to all orders in a weak field expansion.

\subsection{Linear Approximation}

In this subsection, we solve the equations of motion (18) and (19) at the linearized level. The contorsion $S_{a}{ }^{b c}$ can be decomposed to irreducible tensors as

$$
S_{a b c}=V_{b} \delta_{a c}-V_{c} \delta_{a b}+L_{a b c}+H_{a b c}
$$

where $H_{a b c}$ is antisymmetric and $L_{a b c}$ satisfies the relations $L_{[a b c]}=0$ and $L_{a c}^{a}=0$. Using these irreducible tensors, we can linearize (18) and (19) about the flat space, and we obtain

$$
\begin{aligned}
& R_{a b}=(3-d) \partial_{(a} V_{b)}-2(\partial \cdot V) \delta_{a b}+2 \partial^{c} L_{(a b) c} \\
& (1-d) \partial_{[a} V_{b]}+3 \partial^{c} H_{a b c}=0 \\
& \partial_{a}\left(R_{a b}{ }^{c d}+\partial_{a} S_{b}^{c d}-\partial_{b} S_{a}^{c d}\right)=0 .
\end{aligned}
$$

Note that here $\partial_{a}$ represents $\delta_{a}{ }^{\mu} \partial_{\mu}$. The expressions (21) and (22) are the symmetric and antisymmetric part of (18), respectively. As we have discussed below (14), $V_{a}, H_{a b c}$ and $L_{a b c}$ are not gauge fields. Therefore, if we regard them as fundamental fields, there appear negative-norm modes 10 Then, it is natural to regard them as field strengths. As we will see below, this assumption leads to equations of motion of massless fields in string

\footnotetext{
${ }^{10}$ By negative modes we mean fields with odd number of time like index 0 , although we have not started from any field theory action. It is hard to expect such degrees of freedom to posses positive norm when the equation of motion could be derived from a field theory action.
} 
theory at least in the linearized level. For this reason, we consider the following form of solutions:

$$
\begin{aligned}
& V_{a}=\partial_{a} \phi, \\
& H_{a b c}=\partial_{[a} B_{b c]}, \\
& L_{a b c}=0 .
\end{aligned}
$$

Then, the equations of motion reduce to

$$
\begin{aligned}
& R_{a b}=(3-d) \partial_{a} \partial_{b} \phi, \\
& \partial^{2} \phi=0, \\
& \partial^{a} H_{a b c}=0 .
\end{aligned}
$$

Here, the equation (29) is obtained directly from (22). The equation (28) is obtained by substituting (21) to the Bianchi identity $\nabla_{b} R=2 \nabla^{a} R_{a b}$. Using the equation (28), we obtain the equation (27). Using the Bianchi identities $\left.\nabla^{[a} R_{a b} c d\right]=0$ and $\partial_{[a} H_{b c d]}=0$, we can see that (23) gives no other equation.

These equations are the same as the linearized equations of motion for the system of the dilaton and the antisymmetric $B$-field coupled to gravity, which arise naturally from the string theory 11 Note that the conditions (24), (25) and (26) are modified if we include the nonlinear terms. We discuss this point in the next subsection.

Although we have considered solutions which satisfy the conditions (24), (25) and (26), as we will see in the next subsection, and in appendix A, there are many solutions which do not satisfy these conditions. Such solutions are undesirable, because negative norm states can not be removed by the gauge symmetry. We discuss this point further in section 5 .

\subsection{Weak Field Expansion}

In this subsection, we introduce a systematic weak field expansion. We find that for any solution of the linearized equations we can determine higher order corrections order by order. In particular, the solution we discussed in the previous subsection, which looks like the system of the dilaton and the $B$-field coupled to gravity, can be lifted to all orders.

In order to perform the weak field expansion, it is convenient to redefine the fields as

$$
D_{a}=e_{a}{ }^{\mu} \partial_{\mu}+S_{a}^{b c} \mathcal{O}_{b c}
$$

where the quantity $S_{a}^{\prime c d}$ is a sum of the contorsion and the spin connection,

$$
S_{a}^{\prime b c}=S_{a}^{b c}+e_{a}^{\mu} \omega_{\mu}^{b c} .
$$

By expanding $e_{a}{ }^{\mu}$ about the flat space as

$$
e_{a}{ }^{\mu}=\delta_{a}{ }^{\mu}+h_{a}{ }^{\mu},
$$

\footnotetext{
11 An earlier attempt to embed the $B$-field in torsion can be found in [7].
} 
the equations of motion can be written as

$$
\left[D^{a},\left[D_{a}, D_{b}\right]\right]=\left(\partial^{a} f_{a b}^{\mu}-j_{b}{ }^{\mu}\right) \partial_{\mu}+\left(\partial^{a} F_{a b}{ }^{c d}-J_{b}{ }^{c d}\right) \mathcal{O}_{c d}=0
$$

where

$$
f_{a b}{ }^{\mu}=\partial_{a} h_{b}{ }^{\mu}-\partial_{b} h_{a}{ }^{\mu}, \quad F_{a b}{ }^{c d}=\partial_{a} S_{b}^{\prime c d}-\partial_{b} S_{a}^{\prime c d},
$$

and $j_{a}{ }^{\mu}$ and $J_{a}{ }^{c d}$ are the polynomials of $h_{a}{ }^{\mu}$ and $S_{a}^{\prime c d}$. Note that all the indices $a, b, \mu, \nu, \cdots$ are those in the flat space. In particular, the derivative $\partial_{a}$ does not stand for $e_{a}{ }^{\mu} \partial_{\mu}$ but $\delta_{a}{ }^{\mu} \partial_{\mu}$. The quantity $J_{a}{ }^{c d}$ has only the terms of degree two and three in $h_{a}{ }^{\mu}$ and $S_{a}^{\prime c d}$, while the quantity $j_{b}{ }^{\mu}$ has also the linear term;

$$
j_{b}{ }^{\mu}=-\frac{1}{2} \partial^{a} S_{a b}^{\prime \mu}+\partial^{a} S_{b a}^{\prime}{ }^{\mu}-\frac{1}{2} \partial_{b} S_{a}^{\prime a \mu}+\text { terms of degree } 2 \text { and } 3 .
$$

Because $\partial_{\mu}$ and $\mathcal{O}_{c d}$ are independent operators, the equation (33) is equivalent to the following equations:

$$
\begin{aligned}
& \left(\left.\left[D^{a},\left[D_{a}, D_{b}\right]\right]\right|_{\partial}\right)^{\mu}=\partial^{a}{f_{a b}}^{\mu}-j_{b}^{\mu}=0, \\
& \left(\left.\left[D^{a},\left[D_{a}, D_{b}\right]\right]\right|_{\mathcal{O}}\right)^{c d}=\partial^{a} F_{a b}{ }^{c d}-J_{b}{ }^{c d}=0 .
\end{aligned}
$$

These expressions look similar to Yang-Mills theory except that there are extra indices $\mu, c$ and $d$.

Let us solve equations (36) and (37) order by order in a weak field expansion. We expand $h_{a}{ }^{\mu}$ and $S_{a}^{\prime b c}$ as

$$
h_{a}^{\mu}=h_{a}^{(1) \mu}+h_{a}^{(2) \mu}+\cdots, \quad S_{a}^{\prime b c}=S_{a}^{\prime(1) b c}+S_{a}^{\prime(2) b c}+\cdots,
$$

where $h_{a}^{(i) \mu}$ and $S_{a}^{\prime(i) b c}$ represent quantities of $i$-th order. The equations of motion at the $i$-th order are

$$
\begin{gathered}
\left(\left.\left[D^{a},\left[D_{a}, D_{b}\right]\right]^{(i)}\right|_{\partial}\right)^{\mu}=\partial^{a} f_{a b}^{(i) \mu}-j_{b}^{(i) \mu}\left(h^{(1)}, \cdots, h^{(i-1)}, S^{\prime(1)}, \cdots S^{\prime(i-1)}, S^{(i)}\right)=0 \\
\left(\left.\left[D^{a},\left[D_{a}, D_{b}\right]\right]^{(i)}\right|_{\mathcal{O}}\right)^{c d}=\partial^{a} F_{a b}^{(i) c d}-J_{b}^{(i) c d}\left(h^{(1)}, \cdots, h^{(i-1)}, S^{\prime(1)}, \cdots, S^{\prime(i-1)}\right)=0 .
\end{gathered}
$$

Note that $j_{a}^{(i) \mu}$ contains the contribution from $S_{a}^{\prime(i) c d}$, while $J_{a}^{(i) c d}$ does not.

At the lowest order (40) becomes

$$
\partial^{a} F_{a b}^{(1) c d}=0
$$

because $J_{a}^{(1) c d}=0$. This is nothing but the Maxwell equation with the extra indices $c$ and $d$, and can be solved for $S_{a}^{\prime(1) c d}$. By substituting this solution $S_{a}^{\prime(1) c d}$ to (39), we obtain at the lowest order

$$
\partial^{a} f_{a b}^{(1) \mu}=j_{b}^{(1) \mu}\left(S^{\prime(1)}\right)
$$


We can show that the divergence of $j_{b}^{(1) \mu}$ vanishes automatically. Indeed, using (35) and (41), we have

$$
\partial^{b} j_{b}^{(1) \mu}\left(S^{(1)}\right)=-\frac{1}{2}\left(\partial^{2} S_{a}^{\prime(1) a \mu}-\partial^{a} \partial^{b} S_{a b}^{(1) \mu}\right)=-\frac{1}{2} \partial^{a} F_{a b}^{(1) b \mu}=0 .
$$

Therefore, (42) has the same structure as the Maxwell equation with a conserved source except for an extra index $\mu$, and has a solution for $h_{a}^{(1) \mu}$.

In this way, we have solved the equation of motion at the lowest order. We next show that the higher order terms can be determined order by order. Suppose we have solved the equation of motion to the $(n-1)$-th order:

$$
\left[D^{a},\left[D_{a}, D_{b}\right]\right]^{(i)}=0 \quad(i=1, \cdots, n-1) .
$$

Under this assumption, we show that the equation of motion at the $n$-th order,

$$
\left[D^{a},\left[D_{a}, D_{b}\right]\right]^{(n)}=0
$$

or equivalently

$$
\begin{gathered}
\left(\left.\left[D^{a},\left[D_{a}, D_{b}\right]\right]^{(n)}\right|_{\partial}\right)^{\mu}=\partial^{a} f_{a b}^{(n) \mu}-j_{b}^{(n) \mu}\left(h^{(1)}, \cdots, h^{(n-1)}, S^{\prime(1)}, \cdots S^{(n-1)}, S^{\prime(n)}\right)=0 \\
\left(\left.\left[D^{a},\left[D_{a}, D_{b}\right]\right]^{(n)}\right|_{\mathcal{O}}\right)^{c d}=\partial^{a} F_{a b}^{(n) c d}-J_{b}^{(n) c d}\left(h^{(1)}, \cdots, h^{(n-1)}, S^{\prime(1)}, \cdots, S^{\prime(n-1)}\right)=0,
\end{gathered}
$$

also has a solution for $h_{a}^{(n) \mu}$ and $S_{a}^{\prime(n) c d}$. What we need is to show

$$
\begin{aligned}
& \partial^{b} J_{b}^{(n) c d}=0 \\
& \partial^{b} j_{b}^{(n) \mu}=0
\end{aligned}
$$

because if these relations hold, then (46) and (47) can be solved as in the case of (42).

First let us show (48). From (34), $\partial^{a} \partial^{b} F_{a b}^{(n) c d}$ vanish identically. Therefore, we have

$$
\partial^{b} J_{b}^{(n) c d}=-\partial^{b}\left(\partial^{a} F_{a b}^{(n) c d}-J_{b}^{(n) c d}\right) .
$$

By definition, the right hand side of this equation is equal to

$$
-\partial^{b}\left(\left.\left[D^{a},\left[D_{a}, D_{b}\right]\right]^{(n)}\right|_{\mathcal{O}}\right)^{c d}
$$

Noticing that

$$
\begin{aligned}
{\left[\partial^{b},\left[D^{a},\left[D_{a}, D_{b}\right]\right]^{(n)}\right] } & \\
= & \partial^{b}\left(\left.\left[D^{a},\left[D_{a}, D_{b}\right]\right]^{(n)}\right|_{\partial}\right)^{\mu} \cdot \partial_{\mu} \\
\quad & +\partial^{b}\left(\left.\left[D^{a},\left[D_{a}, D_{b}\right]\right]^{(n)}\right|_{\mathcal{O}}\right)^{c d} \cdot \mathcal{O}_{c d}-\left(\left.\left[D^{a},\left[D_{a}, D_{b}\right]\right]^{(n)}\right|_{\mathcal{O}}\right)_{b}{ }_{b}^{c} \partial_{c}
\end{aligned}
$$


we obtain

$$
-\partial^{b}\left(\left.\left[D^{a},\left[D_{a}, D_{b}\right]\right]^{(n)}\right|_{\mathcal{O}}\right)^{c d}=\left(-\left.\left[\partial^{b},\left[D^{a},\left[D_{a}, D_{b}\right]\right]^{(n)}\right]\right|_{\mathcal{O}}\right)^{c d} .
$$

Then, using the equations of motion in the lower orders (44) we have

$$
\left(-\left.\left[\partial^{b},\left[D^{a},\left[D_{a}, D_{b}\right]\right]^{(n)}\right]\right|_{\mathcal{O}}\right)^{c d}=\left(-\left.\left[D^{b},\left[D^{a},\left[D_{a}, D_{b}\right]\right]\right]^{(n)}\right|_{\mathcal{O}}\right)^{c d}
$$

Finally, from the identity $\left[D^{b},\left[D^{a},\left[D_{a}, D_{b}\right]\right]\right]=0$, which is easily derived from the Jacobi identity, the right hand side of (54) is zero. This completes the proof of (48), and hence we can find a solution of (47) for $S_{a}^{\prime(n) c d}$.

Next we show (49). In this case we must use the equation of motion for $S_{a}{ }^{\prime(n) b c}$, in addition to ones for $h^{(i)}$ and $S^{\prime(i)}(i=1, \cdots, n-1)$, because $j_{b}^{(n) \mu}$ depends on $S^{\prime(n)}$ as can be seen from (35). As before, using (34) and (52) we have

$$
\begin{aligned}
\partial^{b} j_{b}^{(n) \mu} & =-\partial^{b}\left(\partial^{a} f_{a b}^{(n) \mu}-j_{b}^{(n) \mu}\right) \\
& =-\partial^{b}\left(\left.\left[D^{a},\left[D_{a}, D_{b}\right]\right]^{(n)}\right|_{\partial}\right)^{c d} \\
& =\left(-\left.\left[\partial^{b},\left[D^{a},\left[D_{a}, D_{b}\right]\right]^{(n)}\right]\right|_{\partial}\right)^{\mu}-\left(\left.\left[D^{a},\left[D_{a}, D_{b}\right]\right]^{(n)}\right|_{\mathcal{O}}\right)_{b}{ }^{\mu}
\end{aligned}
$$

Then, by using (477) and the identity $\left[D^{b},\left[D^{a},\left[D_{a}, D_{b}\right]\right]\right]=0$ the last expression becomes zero, and hence we have (49).

In this way, we can construct the solution order by order. Note that the resemblance to Yang-Mills theory is crucial for the existence of the solution. In appendix A, we discuss how many physical degrees of freedom remain after taking the gauge symmetries into account.

\subsection{Further Analysis for $\phi$}

In section 3.1, we showed that at the linearized level the equations of motion (18) and (19) have a solution composed of the scalar $\phi$ and the rank-two tensor $B_{a b}$. In section 3.2 we showed that any solution at the linearized level can be lifted to a solution of full orders in the weak field expansion. Although the degrees of freedom other than $\phi$ and $B_{a b}$ are not desirable as propagating fields, they are necessary to make full-order solutions. In order to clarify this point, we discuss the difficulty of the following ansatz:

$$
V_{a}=\nabla_{a} \phi, \quad H_{a b c}=0, \quad L_{a b c}=0
$$

We show that (56) gives a rather strong condition on $\phi$ so that $\phi$ cannot be regarded as a scalar field. Note that we impose this condition to all orders. In this ansatz, the Einstein equation (18) becomes

$$
R_{a b}=(3-d) \nabla_{a} \nabla_{b} \phi+(2-d)\left(\nabla_{a} \phi\right)\left(\nabla_{b} \phi\right)-2(\nabla \cdot \phi) \delta_{a b}+(2 d-3)(\nabla \phi)^{2} \delta_{a b} .
$$


Substituting it to the Bianchi identity

$$
2 \nabla^{a} R_{a b}-\nabla_{b} R=0
$$

we obtain

$$
\begin{aligned}
\partial_{a}\{ & \left.(d-1) \nabla^{2} \phi+\left(-d^{2}+d+3\right)(\nabla \phi)^{2}\right\} \\
& +\left(\partial_{a} \phi\right)\left\{(2 d-8)\left(\nabla^{2} \phi\right)+2(3-d)(d-1)(\nabla \phi)^{2}\right\}=0 .
\end{aligned}
$$

This condition is too stringent for $\phi$ to be a scalar field. Indeed, in the weak field expansion, a generic solution of the first order cannot be lifted to all orders.

At the first order (59) reduces to

$$
\partial^{2} \phi^{(1)}=0
$$

and hence $\phi^{(1)}$ is a harmonic function. Then, we determine $e_{a}^{(1) \mu}$ from (57). Using (60), $\nabla^{2} \phi$ becomes a quantity of the second order, and hence the second term in the l. h. s. of (59) is a quantity of the third order. Therefore, at the second order (59) becomes

$$
\left\{(d-1) \nabla^{2} \phi+\left(-d^{2}+d+3\right)(\nabla \phi)^{2}\right\}^{(2)}=c
$$

where $c$ is a constant. Because $\left(\nabla^{2} \phi\right)^{(2)}$ can be rewritten as

$$
\left(\nabla^{2} \phi\right)^{(2)}=\partial^{2} \phi^{(2)}+\left(\nabla^{2} \phi^{(1)}\right)^{(2)}
$$

we can solve $\phi^{(2)}$ in terms of $\phi^{(1)}$ and $e_{a}^{(1) \mu}$, and then we can determine $e_{a}^{(2) \mu}$ from (57). At the third order, (59) becomes

$$
\begin{aligned}
0= & \partial_{a}\left\{(d-1) \partial^{2} \phi^{(3)}+\left(\text { polynomial of } \phi^{(1)}, \phi^{(2)}, e^{(1)}, e^{(2)}\right)\right\} \\
& +\left(\partial_{a} \phi^{(1)}\right)\left\{(2 d-8) \nabla^{2} \phi+2(3-d)(d-1)(\partial \phi)^{2}\right\}^{(2)} \\
= & \partial_{a}\left\{(d-1) \partial^{2} \phi^{(3)}+\left(\text { polynomial of } \phi^{(1)}, \phi^{(2)}, e^{(1)}, e^{(2)}\right)\right\} \\
& +\left(\partial_{a} \phi^{(1)}\right)\left\{(2 d-8) \frac{d^{2}-d-3}{d-1}+2(3-d)(d-1)\right\}\left(\partial \phi^{(1)}\right)^{2} \\
& +c \cdot \frac{2 d-8}{d-1} \partial_{a} \phi^{(1)}
\end{aligned}
$$

where we have used (61) at the second equality. In order for $\phi^{(3)}$ to exist, the second term of the last expression must satisfy the integrability condition, that is, $\left(\partial_{a} \phi^{(1)}\right)\left(\partial \phi^{(1)}\right)^{2}$ must be total derivative. However, this is not the case for generic $\phi^{(1)}$. Therefore, we cannot set $H_{a b c}=0$ and $L_{a b c}=0$; even if we impose $H_{a b c}^{(1)}=L_{a b c}^{(1)}=0, H_{a b c}$ and $L_{a b c}$ appears in higher orders as nonlocal quantities obtained from $\phi$. 


\section{Matrix Model with Additional Terms}

In this section, we study matrix models with a mass term and a cubic term, which were introduced in the context of D-brane action in background fields [8, 9] or as toy models to obtain nontrivial vacua [10, 11]. We analyze the effects of these terms in our interpretation. In section 4.1, we consider matrix models with a mass term. In the paper [3], it was shown that Einstein manifolds are obtained as classical solutions of these models, and for such solutions the coefficient of the mass term plays the role of the cosmological constant. If we introduce a torsion, we find another type of solutions that have constant background of field strength of $B$. In this case, the coefficient of the mass term is given by the field strength. In section 4.2, we consider a three-dimensional matrix model with a cubic term. We find that this model also has the second type of classical solution in which the coefficient of the cubic term can be interpreted as the field strength. In both cases, the antisymmetric part of the torsion equals the field strength of $B$. This result supports the interpretation that the $B$-field is embedded in the torsion.

\subsection{Matrix Model with Mass Term}

In this subsection, we consider the matrix model with a mass term

$$
S=-\frac{1}{4 g^{2}} \operatorname{Tr}\left[A_{a}, A_{b}\right]\left[A^{a}, A^{b}\right]-\frac{\alpha^{2}}{2 g^{2}} \delta_{a b} \operatorname{Tr}\left(A^{a} A^{b}\right),
$$

where $\alpha$ is a parameter with the dimension of mass. We show that this model has two types of solutions without and with torsion. The former consists of the Einstein manifolds and the latter does group manifolds with constant field strength of $B$.

First, let us consider the case where the torsion is absent [3]. For an ansatz $A_{a}=i \nabla_{(a)}$, the equation of motion

$$
\left[A^{a},\left[A_{a}, A_{b}\right]\right]-\alpha^{2} A_{b}=0
$$

becomes

$$
\left[\nabla^{a},\left[\nabla_{a}, \nabla_{b}\right]\right]+\alpha^{2} \nabla_{b}=-\left(R_{b}^{c}-\alpha^{2} \delta_{b}^{c}\right) \nabla_{c}+\left(\nabla^{a} R_{a b}^{c d}\right) \mathcal{O}_{c d}=0
$$

and this is satisfied if the spacetime is an Einstein manifold,

$$
R_{b}^{c}=\alpha^{2} \delta_{b}^{c}
$$

The quantity $\alpha^{2}$ plays the role of a cosmological constant.

We next consider the case in which the torsion is present. For an ansatz $A_{a}=i D_{(a)}$, where $D_{a}$ is a covariant derivative with a torsion, the equation of motion (65) again becomes

$$
\left[D^{a},\left[D_{a}, D_{b}\right]\right]+\alpha^{2} D_{b}=0 .
$$


If the operator $D_{a}$ is proportional to the standard left-invariant derivative on a Lie group $G$, they satisfy the commutation relation of the Lie algebra,

$$
\left[D_{a}, D_{b}\right]=\alpha f_{a b c} D_{c}
$$

where $f_{a b c}$ is the structure constant of $G$. Then the equation of motion (68) is satisfied if $f_{a b c}$ is normalized as

$$
f_{a c d} f_{b} c d=\delta_{a b}
$$

As shown in appendix $\mathrm{B}$, we can interpret $D_{a}$ as the covariant derivative on $G$ with torsion,

$$
D_{a}=\nabla_{a}+S_{a}^{b c} \mathcal{O}_{b c}, \quad S_{a b c}=H_{a b c}=\frac{\alpha}{2} f_{a b c}
$$

Here, the vielbein is defined by

$$
t_{a}=e_{a}^{\mu} \cdot i \alpha g^{-1} \partial_{\mu} g
$$

where $g(x)$ is an element of $G, x$ is a coordinate on $G$ and $t_{a}$ is a generator of $G$, and we have

$$
\begin{aligned}
& R_{a b}{ }^{c d}=\frac{\alpha^{2}}{4} f_{a b e} f^{c d e}=H_{a b e} H^{c d e} \\
& \nabla_{a} S_{b c d}=\nabla_{a} H_{b c d}=0 .
\end{aligned}
$$

The torsion (171) can be regarded as a constant field strength of $B_{a b}$,

$$
H_{a b c}=\nabla_{[a} B_{b c]}
$$

Indeed, from (73) and (74) we obtain the equations

$$
\begin{aligned}
& R_{a}{ }^{c}=H_{a e f} H^{c e f}, \\
& \nabla^{a} H_{a b c}=0,
\end{aligned}
$$

which are nothing but the equations of motion for the metric tensor and the $B$-field 12 . Note that we could set $V_{a}=0$ and $L_{a b c}=0$ in this case while it is not the case when the mass term is absent.

\subsection{Matrix Model with Cubic Term}

In this subsection, we show that the three-dimensional matrix model with a cubic term also has the group manifold $S U(2)$ as a classical solution.

\footnotetext{
12 This set of equations can also be regarded as equations for the graviton, the $B$-field and a constant dilaton with a cosmological constant $\Lambda=\frac{d}{12} \alpha^{2}$.
} 
Let us consider the action

$$
S=-\frac{1}{4 g^{2}} \operatorname{Tr}\left[A_{a}, A_{b}\right]\left[A^{a}, A^{b}\right]+\frac{i \alpha}{3 g^{2}} \epsilon_{a b c} \operatorname{Tr}\left[A^{a}, A^{b}\right] A^{c},
$$

where $\epsilon_{a b c}$ is the structure constant of $S U(2)$. For an ansatz $A_{a}=i D_{(a)}$, the equation of motion is

$$
\left[D_{(a)},\left[D_{(a)}, D_{(b)}\right]\right]-\alpha \epsilon_{b c d}\left[D_{(c)}, D_{(d)}\right]=0
$$

Because the structure constant $\epsilon_{a b c}$ is an invariant tensor of the Lorentz group $\operatorname{Spin}(3)=$ $S U(2)$, we can rewrite (79) as

$$
\left[D_{a},\left[D_{a}, D_{b}\right]\right]-\alpha \epsilon_{b c d}\left[D_{c}, D_{d}\right]=0 .
$$

Therefore, if operators $D_{a}$ satisfy the commutation relation

$$
\left[D_{a}, D_{b}\right]=\alpha \epsilon_{a b c} D_{c}
$$

then $A_{a}=i D_{(a)}$ is a classical solution. As in the case of the previous subsection, this solution describes the group manifold $S U(2) \simeq S^{3}$ with a constant field strength of $B$.

We now comment on dynamical properties of classical solutions. The solution $A_{a}=$ $i D_{(a)}$ can be expressed by a Lie algebra corresponding to the group manifold. Therefore we can discuss dynamical properties of this background using techniques of the noncommutative geometry. On the other hand, the solution without the flux does not form a Lie algebra, and hence the analysis is rather difficult. As a concrete example, we discuss a stability of $S^{3}$ in appendix C.

\section{Conclusions and Discussions}

In the previous paper, we have introduced a new interpretation of the matrix model where matrices act on the space of functions on the principal $\operatorname{Spin}(d)$ bundle over a Riemannian manifold. In this paper, we studied the equation of motion for the vielbein and torsion in the matrix model. In section 3, we interpreted the trace and antisymmetric part of the torsion to be field strengths of the dilaton and the $B$-field, respectively. We found a solution in which only the graviton, dilaton and $B$-field propagate. In general, however, all components of the torsion, including the negative-norm modes, can propagate, and there is no gauge symmetry to remove them. In spite of this difficulty, there are several possibilities that these unnecessary fields disappear from the spectrum. First, recall that higher spin fields couple to the torsion fields in a complicated manner, although we have simply set them to zero. They may give the large mass for the unnecessary degrees of freedom through the interactions. Also there is a possibility that the mass terms of unnecessary fields arise through quantum corrections. If the unnecessary fields disappear through such mechanisms, then our system can reproduce the massless bosonic sector 
of superstring theory. In section 4 , we studied the matrix model with mass and cubic terms, and found solutions with a constant torsion. In this case, the antisymmetric part of the torsion can be interpreted as the constant background of field strength of $B$. This result adds another ground to regard the antisymmetric part of the torsion to be the field strength of the $B$-field.

If unnecessary fields do not disappear from the spectrum dynamically, we may need to impose certain constraints to eliminate them. Although to find a good constraint seems difficult, it might become easier if we first extend the matrix space. One possible way to extend the matrix space is to replace usual manifolds with supermanifolds [13]. This corresponds to consider supermatrices instead of ordinary matrices. Due to this extension, we can implement the local supersymmetry as a subset of the unitary symmetry. In this case, a supermultiplet including the gravity is embedded successfully and the torsion constraint removes unnecessary components of the torsion. In particular, in the case of four dimensions it was shown that the equation of motion of the supermatrix model is consistent with that of $\mathcal{N}=1$ supergravity. This result suggests that a good constraint such as the torsion constraint can be found by extending the matrix space. Although in ten dimensions the superspace formulation is rather complicated and it is not clear whether the torsion constraint fits well to our scheme, the research along this line would be helpful to understand how the spectrum of string theory is derived from the matrix model.

\section{Acknowledgments}

M. H. thanks T. Azeyanagi, T. Azuma and T. Hirata for stimulating discussions and comments. Y. K. is grateful to F. Sugino for helpful discussions. M. H. and Y. K. also thank the Japan Society for the Promotion of Science for financial support. K. F. and M. H. were in part supported by JSPS and French Ministry of Foreign Affairs under the Japan-France Integrated Action Program (SAKURA). M. H. would like to thank CEA/Saclay and Ivan Kostov for hospitality while part of this work was done. This work was also supported in part by a Grant-in-Aid for the 21st Century COE "Center for Diversity and Universality in Physics".

\section{A Physical Degrees of Freedom}

In this section, we consider the physical degrees of freedom of the torsion. We can impose the gauge fixing condition

$$
\partial^{a} S_{a}^{\prime b c}=0, \quad \partial^{a} h_{a}{ }^{\mu}=0
$$

at all orders in the weak field expansion. This condition removes the components along the light-cone direction, $h_{+}{ }^{\mu}$ and $S_{+}^{\prime}{ }^{c d}$. Furthermore, because the equation of motion (36) and (37) can be regarded as the equation of motion for Yang-Mills theory with extra 
indices $\mu, c$ and $d$, we can use the residual symmetries to remove $h_{-}{ }^{\mu}$ and $S_{-}^{\prime}{ }^{c d}$. Therefore, the physical degrees of freedom are

$$
h_{i}{ }^{\mu}, \quad S_{i}^{\prime c d} .
$$

Here, $i$ represents the transverse direction, $i=2,3, \cdots, d-1$. In terms of irreducible tensors with respect to the transverse $S O(d-2)$, they can be decomposed as follows:

$$
\begin{aligned}
& h_{i}^{0}, h_{i}{ }^{1}, h_{i}{ }^{i}, h_{[i j]}, h_{(i j)}-\frac{h_{(k k)}}{d-2} \delta_{i j}, \\
& S_{i}^{\prime 01}, S_{i}^{\prime i 0}, S_{i}^{\prime i 1}, S_{i}^{\prime i j}, S_{(i j)}^{\prime}{ }^{0}-\frac{S_{(k k)}^{\prime}{ }^{0} \delta_{i j}}{d-2}, S_{(i j)}^{\prime}{ }^{1}-\frac{S_{(k k)}^{\prime}{ }^{1} \delta_{i j}}{d-2} \\
& S_{[i j]}^{\prime}{ }^{0}, S_{[i j]}^{\prime}{ }^{1}, S_{[i j k]}^{\prime}, S_{i}^{\prime j k}-\frac{S_{l}^{\prime l k} \delta_{i}{ }^{j}}{d-2}-S_{[i j k]}^{\prime}
\end{aligned}
$$

Unlike in the Yang-Mills theory, fields with indices 0 and 1 are not completely removed. Quantities including 0 as indices, $h_{i}{ }^{0}, S_{i}^{\prime 0 j}$ and $S_{i}^{\prime 01}$, are negative-norm modes.

\section{B Differential Calculus on Lie Group}

In this section, we provide basic results concerning the differential calculus on a Lie group $G$, which are necessary in section 4, For further details and other applications to physics, see [14] for example.

Let $t_{a}$ be the generator of a Lie group $G$ which satisfies the relations

$$
\left[t_{a}, t_{b}\right]=i f_{a b c} t_{c}
$$

The left-invariant derivative $D_{a}$ on $G$ is defined by

$$
\epsilon^{a} D_{a} f(g)=f\left(g\left(1-i \epsilon^{a} t_{a}\right)\right)-f(g)
$$

where $f(g)$ is a function on $G$. From (85), we can easily see that $D_{a}$ satisfies the commutation relation

$$
\left[D_{a}, D_{b}\right]=f_{a b c} D_{c}
$$

By introducing a local coordinate $x, D_{a}$ can be expressed as

$$
D_{a}=v_{a}{ }^{\mu} \partial_{\mu},
$$

where $\partial_{\mu}=\partial / \partial x^{\mu}$ and $v_{a}^{\mu}(x)(a=1, \cdots, \operatorname{dim} G)$ are vector fields on $G$. By taking $f(g)=g, v_{a}{ }^{\mu}$ can be expressed as

$$
t_{a}=v_{a}^{\mu} \cdot i g^{-1}(x) \partial_{\mu} g(x),
$$

where $g(x)$ is the element of $G$ specified by the coordinate $x$. 
Next we identify $v_{a}{ }^{\mu}$ to a vielbein $e_{a}{ }^{\mu}$. From this vielbein, we obtain the standard covariant derivative $\nabla_{a}=e_{a}{ }^{\mu}\left(\partial_{\mu}+\omega_{\mu}{ }^{b c} \mathcal{O}_{b c}\right)$, where $\omega$ is the spin connection. From (88), $\nabla_{a}$ is related to $D_{a}$ as

$$
D_{a}=\nabla_{a}-\omega_{a}^{b c} \mathcal{O}_{b c} .
$$

Then, if we regard the second term of the r.h.s. of (90) as contorsion, $D_{a}$ can be regarded as the covariant derivative with torsion,

$$
D_{a}=\nabla_{a}+S_{a}^{b c} \mathcal{O}_{b c}, \quad S_{a b c}=-\omega_{a b c} .
$$

Combining it with (87), we have

$$
\begin{aligned}
& \mathcal{R}_{a b}{ }^{c d}=0, \\
& S_{a b c}=-\omega_{a b c}=\frac{1}{2} f_{a b c} .
\end{aligned}
$$

We can show the relation $\nabla_{a} f_{b c d}=0$ by using the Jacobi identity:

$$
\begin{aligned}
2 \nabla_{a} S_{b c d} & =\nabla_{a} f_{b c d} \\
& =e_{a}{ }^{\mu} \partial_{\mu} f_{b c d}+\omega_{a b}{ }^{e} f_{e c d}+\omega_{a c}{ }^{e} f_{b e d}+\omega_{a d}{ }^{e} f_{b c e} \\
& =-\frac{1}{2}\left(f_{a b}{ }^{e} f_{e c d}+f_{a c}{ }^{e} f_{b e d}+f_{a d}{ }^{e} f_{b c e}\right) \\
& =0 .
\end{aligned}
$$

By using (15), (16), (92) and (94), the Riemann tensor can be expressed in terms of the structure constant (or equivalently, the torsion) as

$$
\begin{aligned}
R_{a b}{ }^{c d} & =-\nabla_{a} S_{b}{ }^{c d}+\nabla_{b} S_{a}{ }^{c d}+S_{a e}{ }^{c} S_{b}{ }^{e d}-S_{b e}{ }^{c} S_{a}{ }^{e d} \\
& =\frac{1}{4}\left(f_{a e} f_{b}{ }^{e d}-f_{b e}{ }^{c} f_{a}{ }^{e d}\right) \\
& =\frac{1}{4} f_{a b e} f^{c d e} .
\end{aligned}
$$

\section{Group Manifolds in Matrix Model}

In this section, we discuss the covariant derivatives on group manifolds. We begin with the discussion for general group $G$ and then discuss the case of $S U(2)$ in detail.

\section{C.1 Covariant Derivatives on Group Manifolds}

In this subsection, we consider the covariant derivative on a group manifold $G$. As shown in preceding sections, the left-invariant derivative can be identified with the covariant derivative $D_{a}$ with an appropriate field strength of $B$. The Hilbert space $V$ on which $D_{a}$ acts is the set of functions on the principal $\operatorname{Spin}(d)$-bundle over $G$, where $d=\operatorname{dim} G$. 
As we can see from (89), the principal bundle can be covered by a single patch (i.e. the index $a$ in (89) is defined globally), and hence this bundle is the direct product of $G$ and $\operatorname{Spin}(d)$. Therefore, we have

$$
V=C^{\infty}(G \times \operatorname{Spin}(d))=C^{\infty}(G) \otimes C^{\infty}(\operatorname{Spin}(d))
$$

From (86), $D_{a}$ acts on the first component $C^{\infty}(G)$ as the left-invariant derivative, or equivalently as the regular representation. If we denote the regular representation of the generator $t_{a}$ by $T_{a}^{(r e g)}, D_{a}$ can be expressed as

$$
D_{a}=-i T_{a}^{(r e g)} \otimes \mathbf{1}
$$

Using the standard procedure explained in section 2, the index $a$ can be rewritten to that with a parenthesis:

$$
D_{(a)}=R_{(a)}^{b}\left(g^{-1}\right) D_{b}=-i T_{b}^{(r e g)} \otimes R_{(a)}^{b}\left(g^{-1}\right) .
$$

A remark is in order here. Although $D_{a}$ satisfies the commutation relation of the Lie algebra, $D_{(a)}$ does not for $G \neq S U(2)=\operatorname{Spin}(3)$ because the structure constant $f_{a b c}$ is not invariant under the action of $\operatorname{Spin}(d)$. Indeed, the commutation relation of $D_{(a)}$ is

$$
\begin{aligned}
{\left[D_{(a)}, D_{(b)}\right] } & =R_{(a)}{ }^{a^{\prime}}\left(g^{-1}\right) R_{(b)} b^{b^{\prime}}\left(g^{-1}\right)\left[D_{a^{\prime}}, D_{b^{\prime}}\right] \\
& =R_{(a)} a^{a^{\prime}}\left(g^{-1}\right) R_{(b)} b^{\prime}\left(g^{-1}\right) f_{a^{\prime} b^{\prime} c^{\prime}} D_{c^{\prime}} \\
& =R_{(a)} a^{\prime}\left(g^{-1}\right) R_{(b)} b^{\prime}\left(g^{-1}\right) R_{(c)} c^{\prime}\left(g^{-1}\right) f_{a^{\prime} b^{\prime} c^{\prime}} D_{(c)},
\end{aligned}
$$

where $g \in S U(2)$, and the coefficient $R_{(a)}{ }^{a^{\prime}}\left(g^{-1}\right) R_{(b)} b^{b^{\prime}}\left(g^{-1}\right) R_{(c)} c^{c^{\prime}}\left(g^{-1}\right) f_{a^{\prime} b^{\prime} c^{\prime}}$ depends on $g$ unless $G=S U(2)$. In the case of $G=S U(2)$, this coefficient does not depend on $g$,

$$
R_{(a)}^{a^{\prime}}\left(g^{-1}\right) R_{(b)}^{b^{\prime}}\left(g^{-1}\right) R_{(c)} c^{\prime}\left(g^{-1}\right) \epsilon_{a^{\prime} b^{\prime} c^{\prime}}=\epsilon_{a b c},
$$

and $D_{(a)}$ also forms a Lie algebra. Therefore, we can find the explicit form of $D_{(a)}$ in terms of matrices.

\section{C.2 Explicit Form of the Covariant Derivative on $S U(2)$ Group Manifold}

In this subsection, we show the explicit form of the covariant derivative on group manifold $S U(2)$ in terms of matrices. Let $D_{a}^{\prime}$ be the covariant derivative with torsion which is obtained from $D_{a}$,

$$
D_{a}=\nabla_{a}+\frac{1}{2} \epsilon_{a b c} \mathcal{O}_{b c}
$$

by flipping the sign of the torsion:

$$
D_{a}^{\prime}=\nabla_{a}-\frac{1}{2} \epsilon_{a b c} \mathcal{O}_{b c} .
$$


The basic observation is that $D_{(a)}$ and $D_{(a)}^{\prime}$ commute with each other and that both of them satisfy the commutation relation of $S U(2)$ :

$$
\left[D_{(a)}, D_{(b)}^{\prime}\right]=0, \quad\left[D_{(a)}, D_{(b)}\right]=\epsilon_{a b c} D_{(c)}, \quad\left[D_{(a)}^{\prime}, D_{(b)}^{\prime}\right]=-\epsilon_{a b c} D_{(c)}^{\prime} .
$$

Therefore, using an appropriate basis we can write $D_{(a)}$ and $D_{(a)}^{\prime}$ as

$$
\begin{aligned}
& D_{(a)}=-i J_{a}^{(r e g)} \otimes \mathbf{1}, \\
& D_{(a)}^{\prime}=\mathbf{1} \otimes i J_{a}^{(r e g)},
\end{aligned}
$$

where $J^{(r e g)}$ is the regular representation of $S U(2)$. The explicit form of $J^{(r e g)}$ is given by

$$
J^{(r e g)}=\left(\begin{array}{ccccc}
J_{[0]} & & & & \\
& J_{[1 / 2]} \otimes \mathbf{1}_{2} & & & \\
& & \ddots & & \\
& & & J_{[l]} \otimes \mathbf{1}_{2 l+1} & \\
& & & & \ddots
\end{array}\right),
$$

where $J_{[l]}$ stands for the spin- $l$ representation. Using (104), we can express the covariant derivative without torsion $\nabla_{(a)}$ and the Lorentz generator $\mathcal{O}_{a b}$ as

$$
\begin{aligned}
& \nabla_{(a)}=-\frac{i}{2}\left(J_{a}^{(r e g)} \otimes \mathbf{1}-\mathbf{1} \otimes J_{a}^{(r e g)}\right), \\
& \mathcal{O}_{a b}=-\frac{i}{2} \epsilon_{a b c}\left(J_{c}^{(r e g)} \otimes \mathbf{1}+\mathbf{1} \otimes J_{c}^{(r e g)}\right) .
\end{aligned}
$$

In the latter part of this subsection, we discuss whether or not the background $A_{a}=i D_{(a)}$ given in the previous subsection is stable in three-dimensional matrix models. Although the physical interpretation is different, this background is mathematically the same as multiple fuzzy sphere configuration arranged in a concentric pattern, whose dynamical properties have been studied extensively. In the model defined by the action (78), this configuration has flat directions $J_{[l]}^{a} \rightarrow J_{[l]}^{a}+c^{a} \cdot \mathbf{1}_{2 l+1}$, where $c^{a}$ is a constant which represents the translation of each fuzzy sphere, and furthermore, if $c_{a}$ takes a non-zero value, then tachyonic modes arise and the multiple fuzzy sphere configuration decays to a single fuzzy sphere which is described by the irreducible representation [15]. Therefore, the background considered here is unstable in this case. This instability may be removed by introducing supersymmetry or by adding a mass term. For example, the three-dimensional supersymmetric matrix model with the cubic term is considered in [10] and it was shown that multiple fuzzy sphere configuration is BPS if they are arranged in a concentric pattern. Therefore, the configuration (104) would be protected from the quantum correction and be stable, while other configurations which are shifted with nonzero $c^{a}$ are not. In the case that the action has the mass term, it is easy to see that there is no flat direction. In [11, three-dimensional matrix model with the cubic and the positive 
mass term is considered at one-loop level, and it was shown that the multiple fuzzy sphere configuration becomes the true vacuum 13 The multiplicity of each irreducible representation depends on the mass and the coefficient of the cubic term, and it is interesting to investigate whether the configuration (104) can be a true vacuum.

\section{References}

[1] T. Banks, W. Fischler, S. Shenker and L. Susskind, "M theory as a matrix model: a conjecture", Phys. Rev. D55 (1997) 5112; hep-th/9610043.

[2] N. Ishibashi, H. Kawai, Y. Kitazawa and A. Tsuchiya, "A Large $N$ reduced model as superstring”, Nucl. Phys. B498 (1997) 467; hep-th/9612115.

[3] M. Hanada, H. Kawai and Y. Kimura, "Describing curved spaces by matrices," Prog. Theor. Phys. 114 (2006) 1295; hep-th/0508211.

[4] T. Saitou, "Bosonic massless higher spin fields from matrix model," JHEP 0606 (2006) 010; hep-th/0604103.

[5] F. W. Hehl, P. Von Der Heyde, G. D. Kerlick and J. M. Nester, "General Relativity With Spin And Torsion: Foundations And Prospects," Rev. Mod. Phys. 48 (1976) 393.

[6] R. T. Hammond, “Torsion Gravity,” Rept. Prog. Phys. 65 (2002) 599.

[7] J. Scherk and J. H. Schwarz, "Dual Models And The Geometry Of Space-Time," Phys. Lett. B 52, 347 (1974).

[8] R. C. Myers, "Dielectric-branes," JHEP 9912 (1999) 022 hep-th/9910053.

[9] D. Berenstein, J. M. Maldacena and H. Nastase, "Strings in flat space and pp waves from $N=4$ super Yang Mills," JHEP 0204 (2002) 013; hep-th/0202021.

[10] S. Iso, Y. Kimura, K. Tanaka and K. Wakatsuki, "Noncommutative gauge theory on fuzzy sphere from matrix model," Nucl. Phys. B 604 (2001) 121; hep-th/0101102.

[11] T. Azuma, S. Bal and J. Nishimura, "Dynamical generation of gauge groups in the massive Yang-Mills-Chern-Simons matrix model," Phys. Rev. D 72 (2005) 066005; hep-th/0504217.

[12] T. Aoyama, T. Kuroki and Y. Shibusa, "Dynamical generation of non-Abelian gauge group via the improved perturbation theory," hep-th/0608031.

\footnotetext{
${ }^{13}$ In [12, this model was studied further by using the improved perturbation theory, and it was argued that this vacuum is indeed the nonperturbative one.
} 
[13] M. Hanada, H. Kawai and Y. Kimura, "Curved superspaces and local supersymmetry in supermatrix model," Prog. Theor. Phys. 115 (2006) 1003; hep-th/0602210.

[14] E. Braaten, T. L. Curtright and C. K. Zachos, "Torsion And Geometrostasis In Nonlinear Sigma Models," Nucl. Phys. B 260, 630 (1985).

[15] D. P. Jatkar, G. Mandal, S. R. Wadia and K. P. Yogendran, "Matrix dynamics of fuzzy spheres," JHEP 0201 (2002) 039; hep-th/0110172. 